\title{
Review
}

\section{Blunt traumatic aortic injury: CT angiography images and short review of current literature}

\author{
Ferhat Can Piskin ${ }^{1}$, Duygu Ozgul ${ }^{1}$, Yasin Guzel ${ }^{2}$, Erol Huseyin Aksungur ${ }^{1}$ \\ ${ }^{1}$ Department of Radiology and ${ }^{2}$ Department of Cardiovascular Surgery, Cukurova University Faculty of Medicine, Balcali \\ Hospital, Adana, Turkey
}

\begin{abstract}
Blunt traumatic aortic injury in high-energy motor vehicle accidents is a rare but life-threatening condition. Proper treatment after prompt and accurate diagnosis is critical to reduce the death rate. The purpose of this article was to highlight the points to be considered in blunt traumatic aortic injury due to high-energy motor vehicle accident.
\end{abstract}

Key words: Blunt traumatic aortic injury, CT angiography, pseudoaneurysm, aortic rupture, aortic isthmus injury, TEVAR

(Heart Vessels Transplant 2021; 5: doi: 10.24969/hvt.2021.287)

\section{Introduction}

Blunt traumatic aortic injury (BTAI), seen in $3-17 \%$ of high-energy motor vehicle accidents, is a rare but lifethreatening condition $(1,2)$. BTAI is the second most common cause of traumatic death after brain injury with an incidence of $10-20 \%$. Most of the deaths occur before the patient can reach the hospital (3). In addition, BTAl can easily be overlooked without diagnosis, since most of the patients who reach the hospital have non-specific physical examination findings and other accompanying injuries cause symptoms that are more prominent (4).

The purpose of this article was to highlight the points to be considered in blunt traumatic aortic injury due to high-energy motor vehicle accident.

\section{Case one}

A 24-year-old male patient who was brought to the emergency department after a motorcycle accident was evaluated with multiple trauma protocol that covers upper and lower abdomen computed tomography (CT) examinations performed after approximately $70 \mathrm{cc}$ intravenous contrast material injection at a rate of $4 \mathrm{ml} / \mathrm{sec}$ after non-contrast brain, spinal and thorax CT). In the first physical examination, the patient was unconscious, blood pressure was $110 / 85 \mathrm{mmHg}$, heart rate was 88 beats/min and respiratory rate was $25 / \mathrm{min}$.
There were abrasion and ecchymotic areas on the scalp and anterior chest wall.

On CT examination, areas of hemorrhagic contusion in the bifrontal deep white matter in the brain, foci of intraventricular bleeding were detected. In addition, non-contrast thorax CT showed widening of the mediastinal borders and high-density fluid compatible with hematoma around the aortic arch (Fig. 1). Contrast-enhanced arterial phase CT angiography (CTA) was performed with the suspicion of thoracic aortic injury due to the detected findings. On CTA; a fullthickness vessel wall rupture starting from the left subclavian artery orifice at the level of the arcus aorta isthmus and continuing for $5 \mathrm{~cm}$ was observed (Fig. 2). Due to hemodynamic instability, open surgery was preferred over thoracic endovascular aneurysm repair (TEVAR). The patient was taken to open surgery by the cardiovascular surgery team, intubated and the tear in the aorta was repaired with a $26 \mathrm{~mm}$ Dacron graft.

The patient was followed up in the intensive care unit after the operation. On the $16^{\text {th }}$ day of the trauma, the patient general condition deteriorated and blood pressure continued to decrease under maximum inotropic support. Cardiac arrest developed and death occurred despite adequate life support therapy.

Address for correspondence: Ferhat Can Piskin, Department of Radiology, Cukurova University Faculty of Medicine, Balcali Hospital, Adana, Turkey E-mail ferhatcpiskin@gmail.com Mobile: +90 555736 82v83

Received: 19.10.2021 Revised: 13.11.2021 Accepted: 14.11.2021

Copyright @2021 Heart, Vessels and Transplantation 


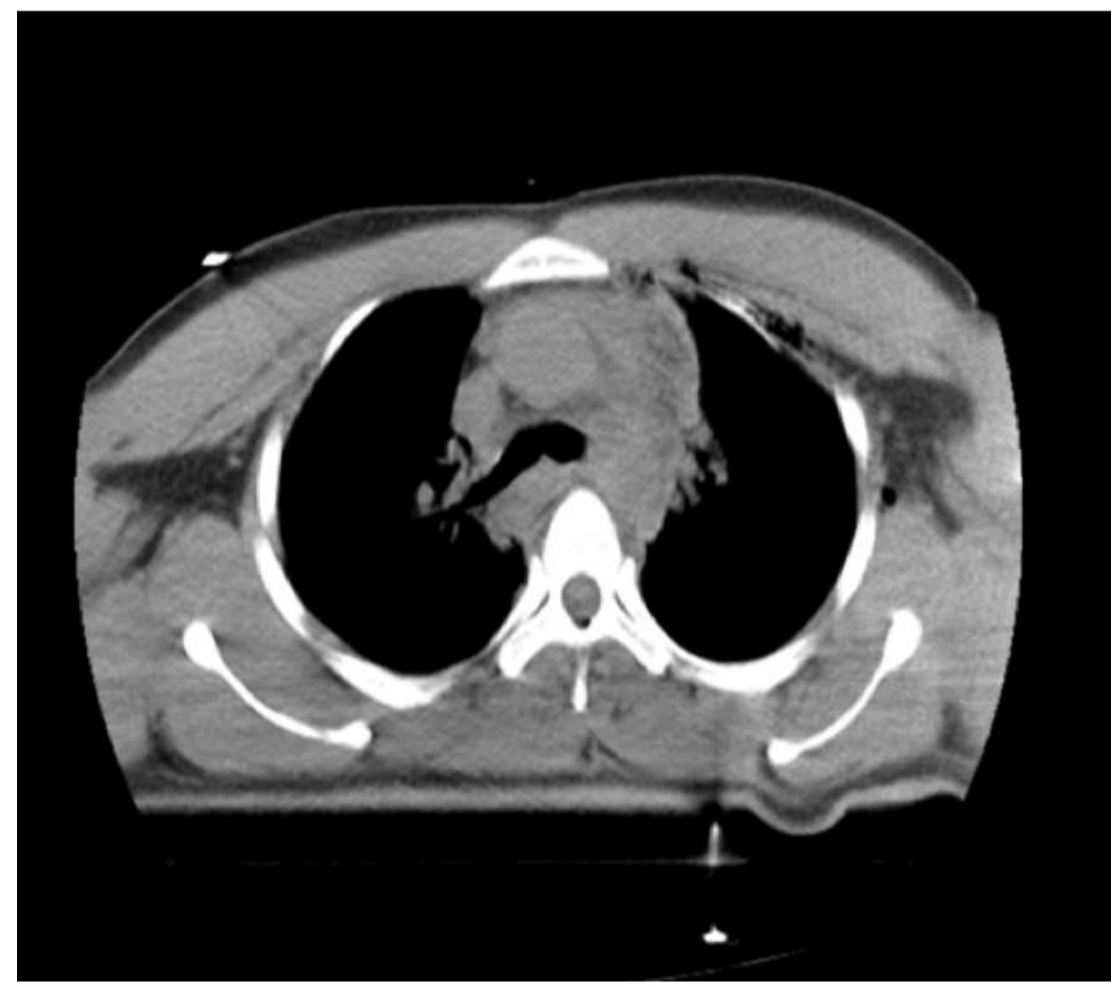

Figure 1. On non-contrast thorax computed tomography (axial images), a hematoma is observed in the mediastinum and around the aorta

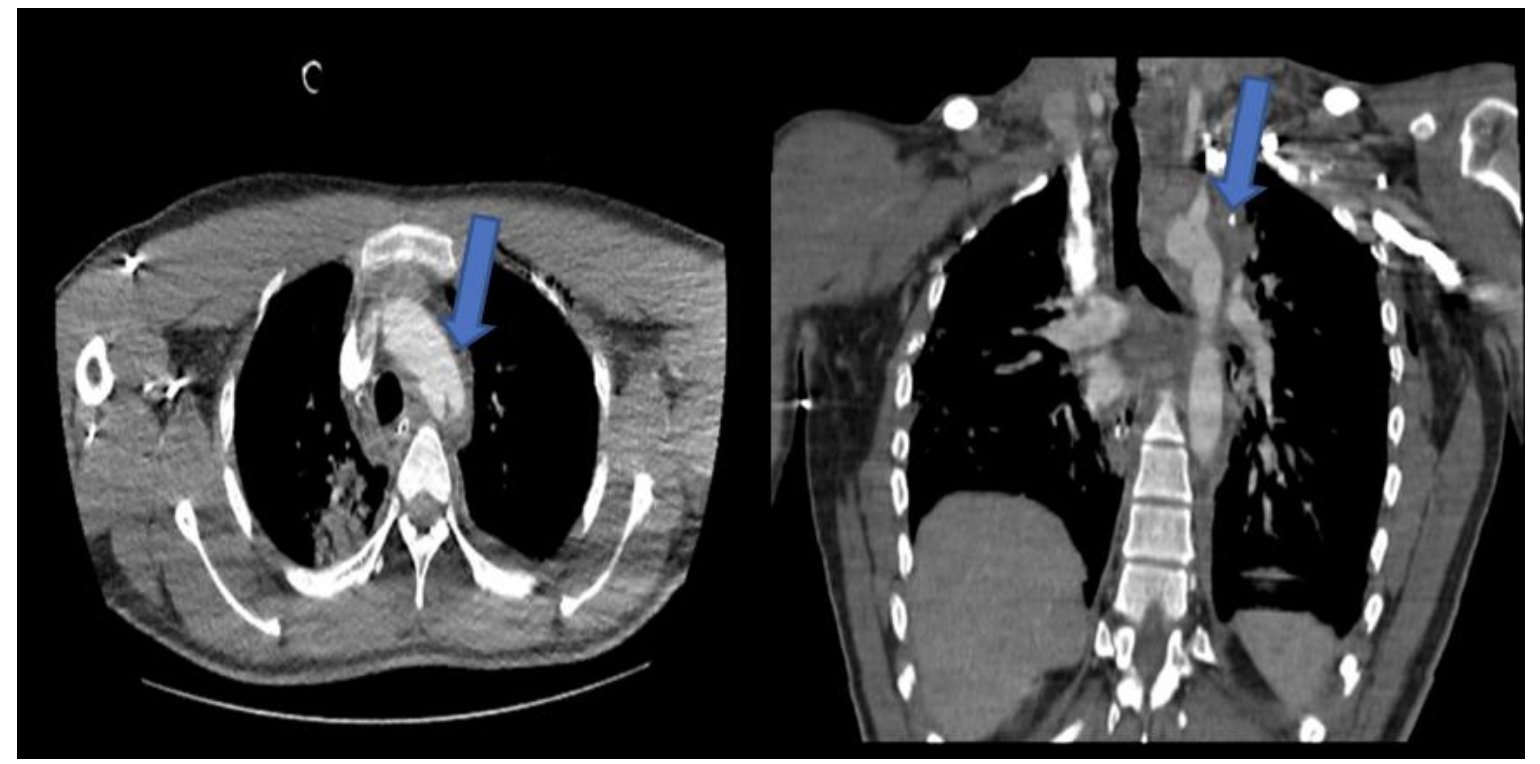

A)

B)

Figure 2. On contrast-enhanced computed tomography of the thorax (axial (a) and coronal (b) images), a complete rupture of the aortic arch at the level of the isthmus is observed (blue arrow). 


\section{Case two}

A 50-year-old male patient who was brought to the emergency department after a traffic accident without any additional disease history was examined upon the detection of multiple, segmented, open fractures in the lower extremity and accompanying chest pain in the initial evaluation. The patient was conscious, blood pressure was 135/85 $\mathrm{mmHg}$, pulse was 77 beats/min, and respiratory rate was $17 / \mathrm{min}$.

Chest examination on non-contrast CT revealed hemopneumothorax on the left and accumulation of fluid consistent with mediastinal high-density hematoma. In addition, a $2 \mathrm{~cm}$ wedge-shaped segmental infarction area, which was thought to have developed on the ground of arterial embolism, was detected in the lower pole of the right kidney in the abdominal examination with intravenous contrast.
CT angiography was performed with suspicion of lower extremity arterial injury and aortic injury. CT angiography showed pseudoaneurysms that started 6 $\mathrm{mm}$ distal from the left subclavian artery orifice and reached $2 \mathrm{~cm}$ in diameter (Fig. 3). In the left tibioperoneal trunk, a filling defect compatible with a partially canalized thrombus was noted. The patient, whose vital signs remained stable during the follow-up but had a decrease in hematocrit values, was treated by the cardiovascular surgery team with TEVAR method. Pseudoaneurysms were repaired with a stent-graft extending from the arcus aorta to the descending aorta. No filling was found in pseudoaneurysms on control examinations. Mediastinal hematoma and left hemothorax findings improved. There was no decrease in hematocrit values during follow-up. The follow-up of the patient, who underwent multiple operations for lower extremity fractures, continues.

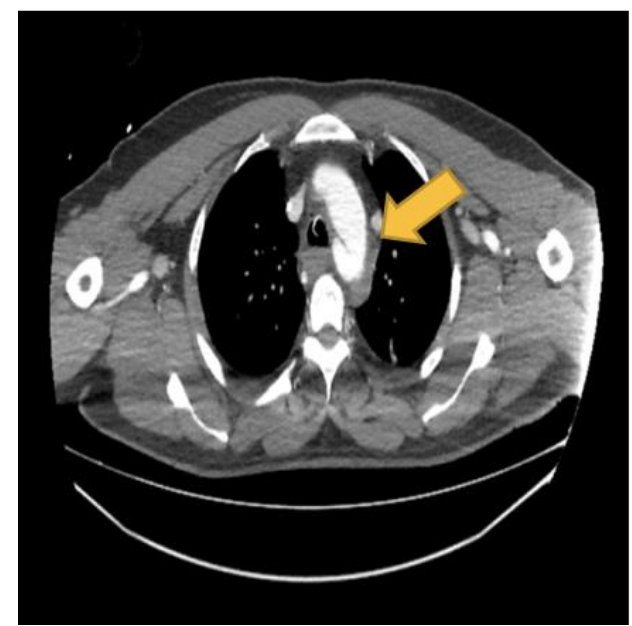

A)

B)

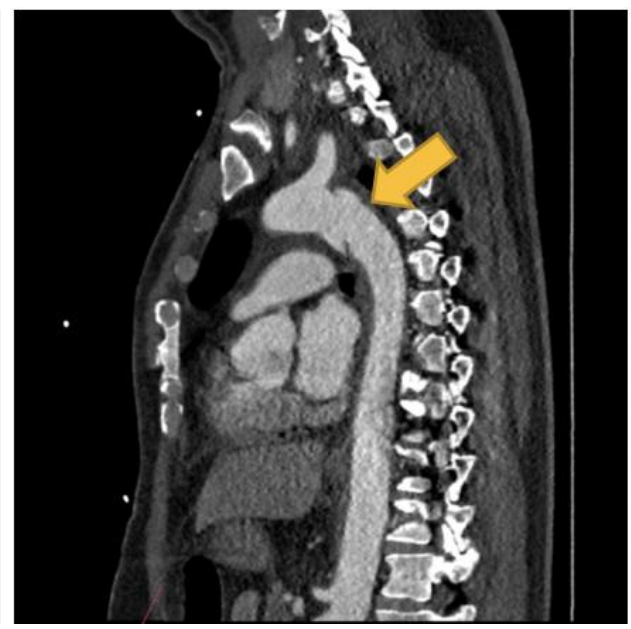

C)

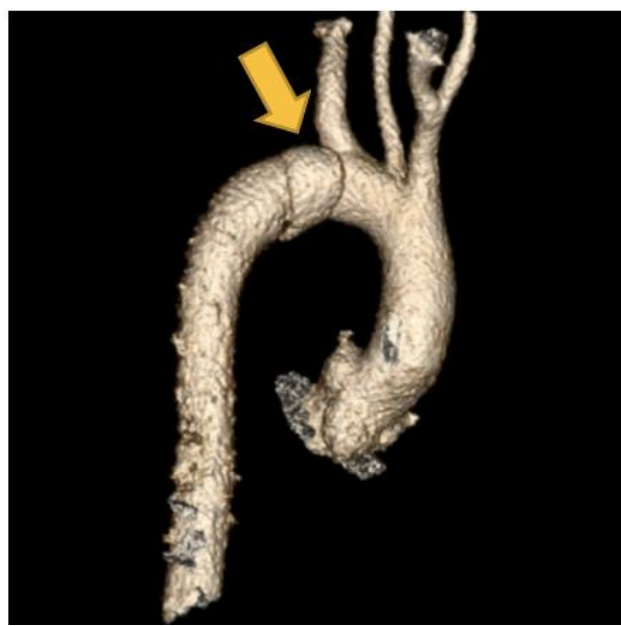

Figure 3. On contrast-enhanced, computed tomography angiography of the thorax (axial (a), coronal (b) and volume rendering (c) images), a saccular structure compatible with a pseudoaneurysm is seen in the isthmus part of the arcus of aorta (yellow arrow)

\section{Discussion}

Undiagnosed and untreated BTAI has a high mortality rate of 90\%, therefore; accurate diagnosis and appropriate treatment for BTAl is so important (4). According to the guideline published by the American Society of Vascular Surgery (SVS) in 2011, based on BTAI radiological imaging findings; aortic injury is classified from minimal injury to severe aortic injury and is handled in 4 four grades (5). Minimal aortic injuries refer to sub-centimetric intima-media injuries that do not show external contour irregularity in the vascular wall (6-8). This group includes small $(<1 \mathrm{~cm})$ intimal tears (grade I) and intramural hematomas (grade II). Significant aortic injuries are defined as pseudoaneurysms (grade III) and aortic rupture (grade IV) (9). 
The aortic isthmus is the most frequently injured part in BTAl, followed by the aortic root and the abdominal aorta at aortic hiatus level. The frequent occurrence of injury at these levels is associated with greater immobility/fixation of these areas compared to other parts of the aorta (10-12). Although the mechanisms of injury to the aorta by blunt trauma are still controversial, rapid deceleration (stretching), osseous compression, shear and hydrostatic forces (water hammer effect) are considered as possible mechanisms. Rapid deceleration (stretching) is an example of the mechanism of injury of the aortic arch, which is subjected to the opposite vertical tension force of the branches of the cranial arch and the caudal heart and intercostal arteries. The osseous compression mechanism explains the aortic injury that occurs as a result of compression between the rigid structures of the sternocostal joint and the vertebral column. The shearing effect is associated with aortic injury because of tensile and torsion forces to which the fixed/immobile points of the aorta, such as the aortic isthmus, are exposed. In the mechanism called hydrostatic forces (water hammer effect), increased intraluminal pressure after blunt trauma is blamed. Although the exact contribution of different forces is not known, convergence/combination of more than one force is considered as a possible theory in the formation mechanism of $\operatorname{BTAI}(13,14)$.

Multiphase CT angiography examination including nonenhanced, arterial and venous phases is sufficient for diagnosis. Non-contrast images are recommended for identifying vessel wall hematoma, arterial phase images for optimal evaluation of the lumen, and venous phase images for revealing possible dissection that shows slow filling and cannot be differentiated from thrombus in arterial phase (15). ECG-triggered examinations are particularly preferred to distinguish injuries at the ascending aorta level from pulsation artifacts. However, ECG-triggered CTA examination is not necessary for this group of patients, because BTAI often develop in the descending thoracic aorta and abdominal aorta (16). In patient management after diagnosis, open surgery or TEVAR can be preferred. Minimal aortic injuries classified as grade I according to SVS can be followed up without treatment by serial imaging methods by providing blood pressure and pulse control (pulse $<70$ 80 beats / $\mathrm{min}$, systolic pressure $<110-120 \mathrm{mmHg}$ ). However, if there are accompanying traumas such as cerebral and spinal injuries that prevent the control of blood pressure and pulse during follow-up, treatment may become mandatory. Low-dose heparin treatment is preferred due to the risk of distal embolism that may develop in the background of turbulent flow. There is no consensus on the treatment of aortic pseudoaneurysms that develop as a result of blunt trauma. There are opinions arguing that pseudoaneurysms less than $1 \mathrm{~cm}$ can also be followed by serial imaging (17-19). In opposing opinions, the treatment of pseudoaneurysms with TEVAR is advocated regardless of their size (19). Treatment is mandatory in significant aortic injuries for grade III and IV according to SVS (5).

Although open surgery is the traditional and most commonly preferred method, the use of TEVAR, which is less invasive, has increased in recent years. During traditional open repair, procedures such as open thoracotomy, one-lung ventilation, aortic clamping, heparinization, which expose the patient group to more risk, are applied and patients need multiple blood product transfusions (21-23). In a 20-year metaanalysis, mortality and paraplegia rates after open surgical repair were reported as 32\% and 9.9\%, respectively (24). Prolongation of aortic clamping time in open surgery may result in paraplegia by affecting spinal cord nutrition on the basis of arterial hypotension and increased cerebrospinal fluid (CSF) pressure (25). However, some patients are not anatomically suitable for TEVAR, which limits the applicability of this method. Due to the lack of sufficient landing zone between the ostium of the left subclavian artery and the lesion that needs to be treated, the ostium of the subclavian artery can be closed by a stent-graft. It is known, that left subclavian artery closure can be tolerated in young patients, but can also result in cerebellar infarction.

There is evidence from meta-analyses that CT examination in the multiple trauma protocol in highenergy trauma patients increases survival compared to the selective imaging method for trauma $(26,27)$. CT evaluation in the multiple trauma protocol generally includes non-contrast brain and cervical vertebra CT, contrast-enhanced thorax and abdomen/pelvis $\mathrm{CT}$, noncontrast multiplanar thoracic and lumbar vertebra CT examinations, although protocols may show different shapes according to medical centers. In our center, thorax imaging is frequently performed without contrast, in the protocol that is widely accepted in adult multiple trauma patients. This situation brings the importance of indirect findings to a critical point in BTAI. 
The aortic isthmus was affected in two presented patients. Both patients were classified with presence of as significant aortic injury. In the first case, there was a grade IV injury, namely aortic rupture, according to SVS, while there was a grade III injury with pseudoaneurysms in the second case. In the first case, the injury resulted in mortality as a result of multiple factors such as concomitant cerebral trauma making blood pressure and pulse control difficult, grade IV blunt traumatic aortic injury and the need for open surgical intervention. In the second case, after the detection of grade III blunt traumatic aortic injury, complete repair was achieved with endovascular treatment applied without delay. Distal embolism due to turbulent flow formed on the basis of pseudoaneurysms resulted in renal segmental infarction and partial canalized thrombus in the infrapopliteal arterial structures of the left lower extremity in the second case. Upon detection of indirect signs of aortic damage (periaortic/mediastinal hematoma, aortic contour irregularity, change in aortic calibration, hemothorax) in both cases, aortic injury was detected without delay by CTA examination, management strategies were defined and patients were referred for treatment.

\section{Conclusion}

Blunt traumatic aortic injury in high-energy motor vehicle accidents is a rare but life-threatening condition. Early diagnosis, clinician-radiologist collaboration with (multidisciplinary approach) and correct treatment significantly reduce mortality. The role of the radiologist is critical in making the diagnosis without wasting time with appropriate imaging tests in order not to miss the indirect findings of blunt traumatic aortic injury. The current classification of blunt traumatic aortic injuries, regulated by the guideline published by SVS, greatly influences patient management and treatment planning. In terms of treatment methods, TEVAR has been considered as a frequently preferred method in recent years.

Ethics: Informed consent was obtained from the patients's family before all procedures Peer-review: External and internal Conflict of interest: None to declare Authorship: F.C.P., D.O., Y.G. and E.H.A. are equally contributed to patients' management and preparation of manuscript

Acknowledgement and funding: None to declare

\section{References}

1.Fabian TC, Richardson JD, Croce MA, Smith JS Jr, Rodman G Jr, Kearney PA, et al. Prospective study of blunt aortic injury: Multicenter Trial of the American Association for the Surgery of Trauma. J Trauma 1997; 42: 374-80.

2.Frick EJ, Cipolle MD, Pasquale MD, Wasser TE, Rhodes $M$, Singer RL, et al. Outcome of blunt thoracic aortic injury in a level I trauma center: an 8-year review. J Trauma 1997; 43: 844-51.

3.Tefft B. Rates of motor vehicle crashes, injuries and deaths in relation to driver United States, 2014-2015. AAA Foundation for Traffic Safety, 2017.

4.Parmley Lf, Mattıngly Tw, Manıon Wc, Jahnke Ej Jr. Nonpenetrating traumatic injury of the aorta. Circulation 1958; 17: 1086-101.

5.Lee WA, Matsumura JS, Mitchell RS, Farber MA, Greenberg RK, Azizzadeh A, et al. Endovascular repair of traumatic thoracic aortic injury: clinical practice guidelines of the Society for Vascular Surgery. J Vasc Surg 2011; 53: 187-92.

6.Heneghan RE, Aarabi S, Quiroga E, Gunn ML, Singh N, Starnes BW. Call for a new classification system and treatment strategy in blunt aortic injury. J Vasc Surg 2016; 64: 171-6.

7.Quiroga E, Starnes BW, Tran NT, Singh N. Implementation and results of a practical grading system for blunt thoracic aortic injury. J Vasc Surg 2019 ; 70: 1082-8.

8.Starnes BW, Lundgren RS, Gunn M, Quade S, Hatsukami TS, Tran NT, et al. A new classification scheme for treating blunt aortic injury. J Vasc Surg 2012; 55: 47-54.

9.Azizzadeh A, Keyhani K, Miller CC 3rd, Coogan SM, Safi HJ, Estrera AL. Blunt traumatic aortic injury: initial experience with endovascular repair. J Vasc Surg 2009; 49: 1403-8. 10.Teixeira PG, Inaba K, Barmparas G, Georgiou C, Toms C, Noguchi TT, et al. Blunt thoracic aortic injuries: an autopsy study. J Trauma 2011; 70: 197-202.

11.Burkhart HM, Gomez GA, Jacobson LE, Pless JE, Broadie TA. Fatal blunt aortic juries: a review of 242 autopsy cases. J Trauma 2001; 50: 113-5.

12.Feczko JD, Lynch L, Pless JE, Clark MA, McClain J, Hawley DA. An autopsy case review of 142 nonpenetrating (blunt) injuries of the aorta. J Trauma 1992; 33: 846-9. 
13.Richens D, Field $M$, Neale $M$, Oakley $C$. The mechanism of injury in blunt traumatic rupture of the aorta. Eur J Cardiothorac Surg 2002; 21: 288-93.

14.Neschis DG, Scalea TM, Flinn WR, Griffith BP. Blunt aortic injury. N Engl J Med 2008; 359: 1708-16.

15.Mouawad NJ, Paulisin J, Hofmeister S, Thomas MB. Blunt thoracic aortic injury - concepts and management. J Cardiothorac Surg 2020; 15: 62.

16.Cullen EL, Lantz EJ, Johnson CM, Young PM. Traumatic aortic injury: CT findings, mimics, and therapeutic options. Cardiovasc Diagn Ther 2014; 4: 238-44.

17.Tanizaki S, Maeda S, Matano $H$, Sera $M$, Nagai $H$, Nakanishi $T$, Ishida $H$. Blunt thoracic aortic injury with small pseudoaneurysm may be managed by nonoperative treatment. J Vasc Surg 2016; 63: 341-4.

18.Gandhi SS, Blas JV, Lee S, Eidt JF, Carsten CG 3rd. Nonoperative management of grade III blunt thoracic aortic injuries. J Vasc Surg 2016; 64: 1580-6.

19.Harris DG, Rabin J, Bhardwaj A, June AS, Oates CP, Garrido $D$,. Nonoperative management of traumatic aortic pseudoaneurysms. Ann Vasc Surg 2016; 35: 7581.

20.Kapoor H, Lee JT, Orr NT, Nisiewicz MJ, Pawley BK, Zagurovskaya M. Minimal aortic injury: mechanisms, imaging manifestations, natural history, and management. Radiographics 2020; 40: 1834-47.

21.Hoffer EK, Karmy-Jones R, Bloch RD, Meissner MH, Borsa JJ, Nicholls SC, et al. Treatment of acute thoracic aortic injury with commercially available abdominal aortic stent-grafts. J Vasc Interv Radiol 2002; 13: 103741.

22.Tang GL, Tehrani HY, Usman A, Katariya K, Otero C, Perez $\mathrm{E}$, et al. Reduced mortality, paraplegia, and stroke with stent graft repair of blunt aortic transections: a modern meta-analysis. J Vasc Surg 2008; 47: 671-5.

23.Xenos, E.S., Abedi NN, Davenport DL, Minion DJ, Hamdallah O, Sorial EE, et al. Meta-analysis of endovascular vs open repair for traumatic descending thoracic aortic rupture. J Vasc Surg 2008; 48: 1343-51. 24.von Oppell UO, Dunne TT, De Groot MK, Zilla P. Traumatic aortic rupture: twenty-year meta-analysis of mortality and risk of paraplegia. Ann Thorac Surg 1994; 58: 585-93.

25.Katz NM, Blackstone EH, Kirklin JW, Karp RB. Incremental risk factors for spinal cord injury following operation for acute traumatic aortic transection. J Thorac Cardiovasc Surg 1981; 81: 669-74.

26.Caputo ND, Stahmer C, Lim G, Shah K. Whole-body computed tomographic scanning leads to better survival as opposed to selective scanning in trauma patients: a systematic review and meta-analysis. J Trauma Acute Care Surg 2014; 77: 534-9.

27.Chidambaram S, Goh EL, Khan MA. A meta-analysis of the efficacy of whole-body computed tomography imaging in the management of trauma and injury. Injury 2017; 48: 1784-93. 


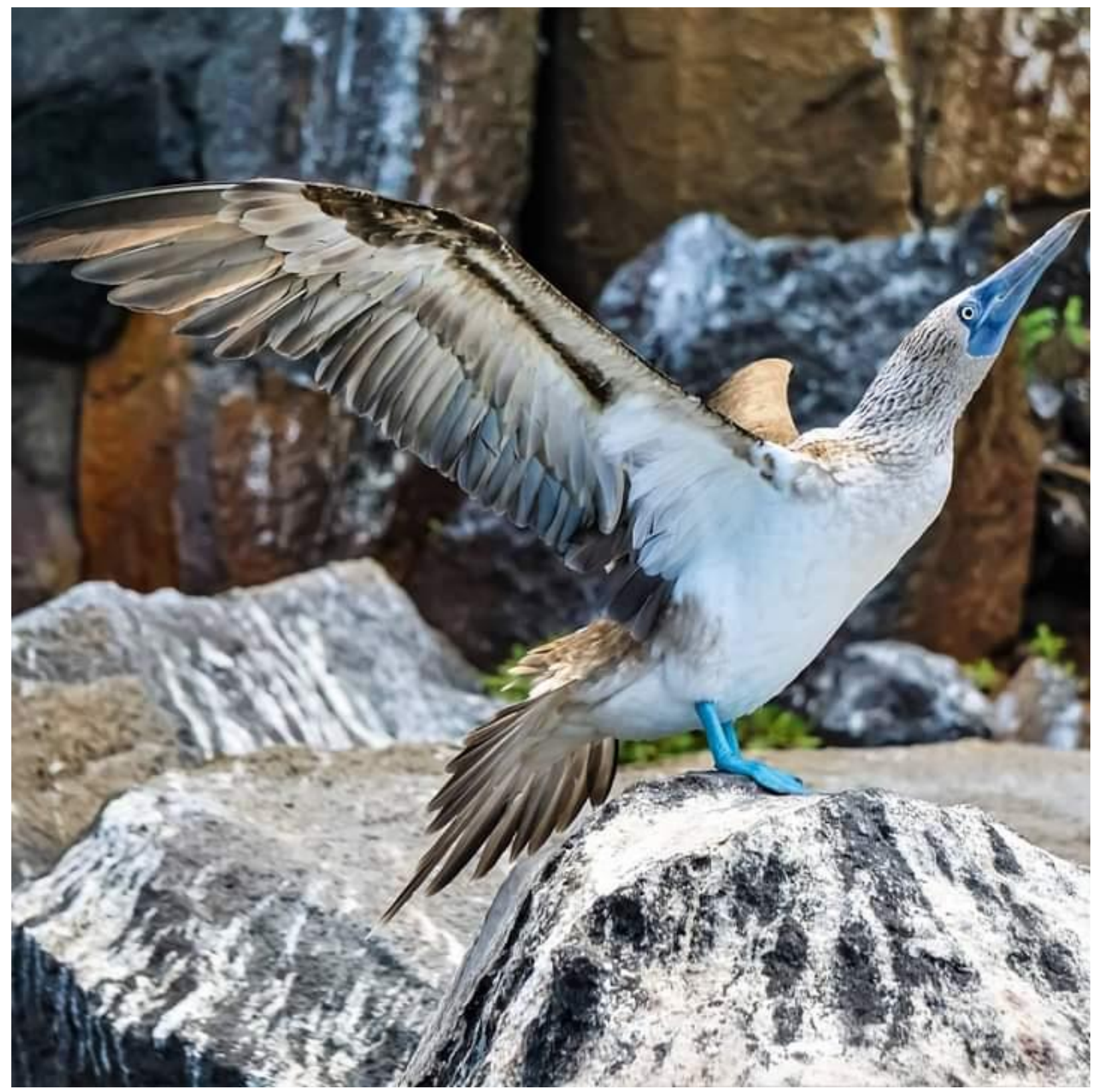

Blue-footed booby (Sula nebouxii), Galapagos Ireland, 2021. This smart bird is distinguished by blue foot due to a fish diet rich of carotenoids, it is an excellent divers and is called booby (Spanish bobo -foolish) because of the awkward walking (www.nationalgeographic.com, www.latin2travel.com). Anna Zelek, Washington, USA. 